\title{
A Dual Model-Free Control of Underactuated Mechanical Systems, Application to The Inertia Wheel Inverted Pendulum
}

\author{
S. Andary, A. Chemori, M. Benoit and J. Sallantin
}

\begin{abstract}
This paper deals with a new method allowing recent model-free control technique to deal with underactuated mechanical systems for stable limit cycles generation. A model-free controller is designed in order to track some parametrized reference trajectories. A second model-free controller is then designed using trajectories' parameters as control inputs in order to stabilize the internal dynamics. The proposed method is applied to a real underactuated mechanical system: the inertia wheel inverted pendulum. Numerical simulations as well as real-time experiments are presented showing the effectiveness of the proposed control method and its robustness toward external disturbances.
\end{abstract}

\section{INTRODUCTION}

Model-free control strategies has been recently proposed in [1], [2] resulting in a breakthrough in nonlinear control. This technique is based on recent results on fast estimation and identification of nonlinear signals [3], [4]. The control scheme is based on local linear approximation of the controlled system dynamics which is valid for a small time window. This approximation is updated in an online fashion thanks to a fast estimator. The control law proposed consists in a PID controller augmented with compensating terms provided by the online estimation of the system dynamics. The overall controller is called $i$ PID (intelligent PID) controller. Comparison of such a controller with classical PID controller can be found in [5]. The main advantage of this control strategy is that it doesn't require neither prior knowledge of the system dynamics, nor complex parameters tuning. It is therefore easy to build a controller for an unknown system. Modelfree control has already been utilized in a number of most practical case studies (see [6], [7], [8]).

Although this control method is smart, and has been applied to resolve many control problems, it has however some drawbacks. Aside it's dependancy to quality of sensors and sampling frequency on which relies the fast local estimation, model-free control is not curently adapted to the control of underactuated systems. Such systems are often characterized by unstable internal dynamics. In this paper we focus on stable limit cycles generation for underactuated mechanical systems. A mechanical system with less actuators than degrees of freedom is said to be underactuated and unfortunately a vast majority of these

S. Andary, A. Chemori, M. Benoit and J. Sallantin are with LIRMM, Univ. Montpellier 2 - CNRS, 161 rue Ada, 34392 Montpellier, France \{andary, chemori, Michel.Benoit, js\}@lirmm.fr systems are non-minimum phase. Therefore model-free control technique cannot be applied as it has initially been proposed. Some efforts have been made for two particular cases of underactuated systems: the ball and beam [9] (where the dynamics of the beam has not been taken into account) and the Planar Vertical Take Off and Landing (PVTOL) aircraft [10] where the adressed control problem is limited to state regulation.

The main contribution of this paper is to design a new control scheme based on model-free control approach to deal with underactuated mechanical systems for stable limit cycle generation. The proposed method is applied to an inertia wheel pendulum with real-time experimental results.

The inertia wheel pendulum was introduced in [11] as a benchmark system for nonlinear control of underactuated mechanical systems. The controller proposed by the authors consists of two separates control laws: a swingup controller based on partial feedback linearisation and energy shaping and a linear balancing controller. In [12], swing-up and balance of the pendulum is achieved with a single controller using interconnection and damping assignment wich can be seen as a generalisation of controlled Lagrangians method. This mechanical system still shows recent interest among nonlinear control community. In [13], strong damping force on the inertia wheel is taken into account in the design of the controller. Stabilization is achieved via nested saturation based controller. [14] solves the limit cycles generation problem on the inertia wheel pendulum using virtual holonomic constraint. Real-time experiments are carried out showing the robustness of the approach. In [15], collocated partial feedback linearization is performed to exhibit the nonlinear core subsystem wich is stabilized using an implicit control. The remaining subsystem is stabilized using multiple sliding mode technique. In our previous work (see [16], [17]), non-collocated partial feedback linearization is used; this gives arise to an unstable internal dynamics, which is stabilized using trajectory optimization and model based error estimation. All the mentionned techniques require knowledge of the system dynamics and parameters, furthermore only [14], [16] present real-time experiments.

In order to achieve stable limit cycles on all coordinates of the inertia wheel pendulum, we first design a family of parametrized periodic trajectories for the pendulum angle. Thoses trajectories are then tracked using control inputs thanks to a classical model-free controller. Since 
the system is underactuated (non-minimum phase), the internal dynamics of the system is unstable. Stable limit cycles on the actuated coordinate are generated through the control of trajectories' parameters. To achieve this control, we propose an other model-free controller using actuated coordinate as output and trajectories' parameter as input. Note that this control scheme can be extended to regulation control by carefully choosing apropriate trajectories.

The rest of the paper is organised as follow. Section II presents the proposed dual model-free controller. The experimental testbed, numerical simulations as well as real-time experiments are presented in Section III. Finally, conclusion and future work are discussed in the Section IV.

\section{DuAl MODEL-FREE CONTROLleR}

In our case, we are interrested in stable limit cycles generation for under-actuated mechanical systems which are generally nonlinear and non-minimum phase. In order to simplify our presentation, we focus on 1-input 2-degree of freedom mechanical systems which are the minimum dimensions for a system to be underactuated. The dynamics of such systems takes the following lagrangian matrix form [18], [19]:

$$
M(q) \ddot{q}+H(q, \dot{q})+G(q)=R u
$$

where $M \in \mathbb{R}^{2 \times 2}$ is the inertia matrix of the system, $q \in \mathbb{R}^{2}$ is the vector of generalized coordinates. $\dot{q}, \ddot{q} \in$ $\mathbb{R}^{2}$ are respectively their first and second derivatives. $H \in \mathbb{R}^{2}$ is a vector containing centrifugal and Coriolis forces terms and $G \in \mathbb{R}^{2}$ is a vector of gravitational terms. $u \in \mathbb{R}$ is the control input and $R \in \mathbb{R}^{2 \times 1}$ is a matrix distributing the effects of $u$ on the generalized coordinates. Using a suitable partition $q=\left[q_{a}, q_{n a}\right]^{T}$ of the vector of generalized coordinates where $q_{a}$ is the actuated coordinate and $q_{n a}$ is the unactuated one, equation (1) can be rewritten as:

$$
\begin{aligned}
& m_{11}(q) \ddot{q}_{a}+m_{12}(q) \ddot{q}_{n a}+h_{1}(q, \dot{q})+g_{1}(q)=u \\
& m_{21}(q) \ddot{q}_{a}+m_{22}(q) \ddot{q}_{n a}+h_{2}(q, \dot{q})+g_{2}(q)=0
\end{aligned}
$$

with :

$$
M=\left[\begin{array}{ll}
m_{11} & m_{12} \\
m_{21} & m_{22}
\end{array}\right] ; \quad H=\left[\begin{array}{l}
h_{1} \\
h_{2}
\end{array}\right] ; \quad G=\left[\begin{array}{l}
g_{1} \\
g_{2}
\end{array}\right]
$$

We suppose that the state of the system is the vector $\left[\begin{array}{llll}q_{a} & q_{n a} & \dot{q}_{a} & \dot{q}_{n a}\end{array}\right]^{T}$.

\section{A. Basic principle of the proposed method}

Our goal is to generate stable limit cycles on both actuated and unactuated coordinates. We first define a family of $p$-parametrized $\tau$-periodic reference trajectories $q_{n a}^{*}(p, \tau, t)$ for the unactuated coordinate. Those trajectories have the same boundary conditions for all $p$ values, allowing the controller to switch from one trajectory to another while the overall trajectory remains smooth. Thanks to the dynamic coupling existing between the

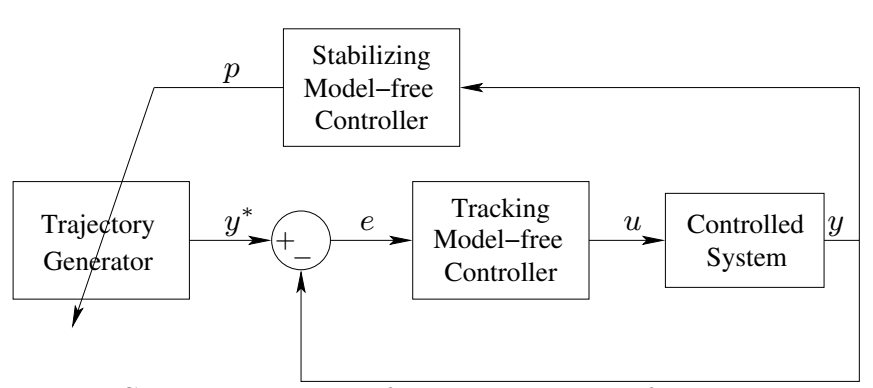

Fig. 1: Schematic view of the dual model-free controller.

actuated and unactuated coordinates, it is possible to control directly the unactuated coordinate using the control input $u$ (i.e. the torque on the actuated coordinate) which allows those trajectories to be tracked on the unactuated coordinate $q_{n a}$ using the control input $u$. Indeed, the dynamics (2)-(3) can be rewritten in a form which explicits the relation between unactuated coordinate and control input. First, equation (2) is solved for $\ddot{q}_{a}$ (for clarity reason the dependancy in $q$ and $\dot{q}$ of the terms involved is omitted in the notation):

$$
\ddot{q}_{a}=m_{11}^{-1}\left(-m_{12} \ddot{q}_{n a}-h_{1}-g_{1}+u\right)
$$

Injecting this solution in equation (3) leads to:

$$
\bar{m}_{2} \ddot{q}_{n a}+\bar{h}_{2}+\bar{g}_{2}=-m_{21} m_{11}^{-1} u
$$

where $\bar{m}_{2}=m_{22}-m_{21} m_{11}^{-1} m_{12}, \bar{h}_{2}=h_{2}-m_{21} m_{11}^{-1} h_{1}$ and $\bar{g}_{2}=g_{2}-m_{21} m_{11}^{-1} g_{1}$.

A model-free controller can then be designed to perform the tracking of reference trajectories on unactuated coordinate using the control input $u$. In order to stabilize the internal dynamics of the closed-loop system (i.e. the inertia wheel dynamics) and to generate stable limit cycles on both coordinates, a second controller is designed. This second controller therefore takes the reference trajectory parameter $p$ as control input, and uses the actuated coordinate as output. At the end of each period (of the cyclic reference trajectory), the second controller chooses the right trajectory parameter $p$ in order to stabilize the actuated coordinate. The choosen parameter $p$ fixes the reference trajectory used by the first controller for the duration of the whole next period. The overall control scheme is illustrated in the blockdiagram of Fig. 1.

\section{B. Parametrized reference trajectories generation}

The first step of the proposed framework is to generate parametrized reference trajectories $q_{n a}^{*}(p, \tau, t)$ to be tracked on the unactuated coordinate. Those trajectories must fulfil some conditions. First of all, they have to be continuous, derivable and periodic in order to generate limit cycles. That leads us to design oscillatory shaped trajectories which are splitted in half period, where we will use symmetry to generate the whole cycle. The parametrization of these trajectories must allow the controller to update the parameter $p$ (which corresponds to the time at which the trajectory $q_{n a}^{*}$ crosses zero) during 
tracking while the overall trajectory remains smooth. This leads to some initial and final conditions of each half period part. That is for a given period $\tau$ and amplitude $2 A$ :

$$
\forall p \in \mathcal{P},\left\{\begin{array}{l}
q_{n a}^{*}(p, \tau, 0)=q_{n a}^{*}(p, \tau, \tau)=A \\
q_{n a}^{*}(p, \tau, \tau)=A \\
\dot{q}_{n a}^{*}(p, \tau, 0)=\dot{q}_{n a}^{*}\left(p, \tau, \frac{\tau}{2}\right)=\dot{q}_{n a}^{*}(p, \tau, \tau)=0
\end{array}\right.
$$

for some domain $\mathcal{P} \subset \mathbb{R}$ (choosen to keep an oscillatory shape). We propose to use a six-degree polynomial function parametrized with $p$ such that:

$$
\forall p \in \mathcal{P}, q_{n a}^{*}(p, \tau, t=p)=0
$$

\section{Proposed dual model-free controller}

The design of the reference trajectories tracking controller is based on a model-free controller. The unactuated coordinate nonlinear dynamics (5) is replaced by the local model according to model-free control principle:

$$
\ddot{q}_{n a}=F_{1}+\alpha_{1} u
$$

where the constant parameter $\alpha_{1}$ is a design parameter. $F_{1}$ captures the nonlinearities in the unactuated coordinate dynamics and is updated according to the following equation at each sample time:

$$
\left[F_{1}(k)\right]_{e}=\left[\ddot{q}_{n a}(k)\right]_{e}-\alpha_{1} u(k-1)
$$

The notation [.] $]_{e}$ is the estimated value.

The tracking controller is obtained using numericaly computed value of $F_{1}$ in (9) using the first model-free controller $(i$-PID):

$$
u=\frac{1}{\alpha_{1}}\left(-F_{1}+\ddot{q}_{n a}^{*}(p, \tau, t)+K_{p 1} e+K_{i 1} \int e+K_{d 1} \dot{e}\right)
$$

with PID gains $K_{p 1}, K_{i 1}, K_{d 1}$ which can be chosen using poles placement technique since nonlinearities of the system dynamics are supposed to be compensated by the $F_{1}$ term. The unactuated coordinate follows therefore the desired periodic trajectories $q_{n a}^{*}(p, \tau, t)$.

The parameter $p$, used in the tracking control input (10), is constant over half a period $\forall t \in\left[k \frac{\tau}{2}(k+1) \frac{\tau}{2}[\right.$, $(k \in \mathbb{N})$ and is updated at the end of each half period at time $(k+1) \frac{\tau}{2}$ by the second controller (13). The unknown nonlinear dynamics of the actuated coordinate is replaced by the local discrete model:

$$
\Delta_{\tau} v_{a}=F_{2}+\alpha_{2} p
$$

where $\Delta_{\tau} v_{a}=\dot{q}_{a}\left(k \frac{\tau}{2}\right)-\dot{q}_{a}\left((k-1) \frac{\tau}{2}\right)$ is the variation of actuated articulation velocity $v_{a}=\dot{q}_{a}$ measured between half periods and the constant $\alpha_{2}$ is a design parameter. The value of $F_{2}$ is updated at the end of each half cycle $\left(t=k \frac{\tau}{2}\right.$ for $\left.k \in \mathbb{N}\right)$ using the principle of model-free control:

$$
\left[F_{2}\left(k \frac{\tau}{2}\right)\right]_{e}=\left[\Delta_{\tau} \dot{q}_{a}\left(k \frac{\tau}{2}\right)\right]_{e}-\alpha_{2} p\left((k-1) \frac{\tau}{2}\right)
$$

Note that the actuated coordinate dynamics whithin a half cycle $t=\left[k \frac{\tau}{2}(k+1) \frac{\tau}{2}\right]$ is not taken into account in this local model since we only aim at limit

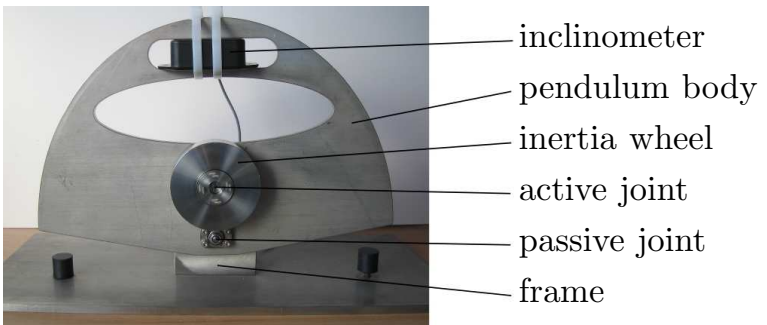

Fig. 2: The inertia wheel inverted pendulum

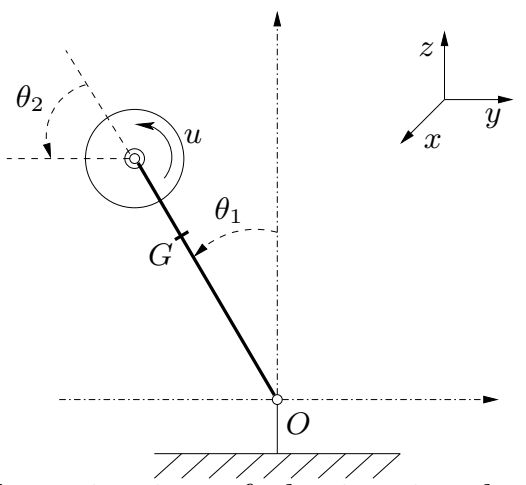

Fig. 3: Schematic view of the inertia wheel inverted pendulum: the joint between the frame and the beam is unactuated (passive), while the one between the beam and the inertia wheel is actuated (active).

cycle generation and therefore it is only required that the actuated coordinate trajectory be periodic. In other words, the aim of the second controller is to bring the actuated coordinate to a fixed desired state $\left(q_{a}^{d}, \dot{q}_{a}^{d}\right)$ at the end of each half period, ensuring periodicity of the actuated coordinate trajectory and therefore limit cycles generation. The second model-free controller updates the trajectory parameter $p$ according to the following formula:

$p=\frac{1}{\alpha_{2}}\left(-F_{2}+K_{p 2}\left(q_{a}^{d}-q_{a}\right)+K_{i 2} \int\left(q_{a}^{d}-q_{a}\right)+K_{d 2}\left(\dot{q}_{a}^{d}-\dot{q}_{a}\right)\right)$

where $K_{p 2}, K_{i 2}, K_{d 2}$ are the PID gains. Notice that since the desired state for actuated coordinate $\left(q_{a}^{d}, \dot{q}_{a}^{d}\right)$ is constant, the $\left(\Delta_{\tau} \dot{q}_{a}\right)^{d}$ term is zero and is then ommited.

\section{Application: the inertia WhEel inverted PENDULUM}

The proposed control scheme is applied to an underactuated mechanical system: the inertia wheel inverted pendulum (cf. Fig. 2), which consists of an inverted pendulum equipped with a rotating wheel. The joint between the pendulum body and the frame is unactuated whereas the joint between the beam and the wheel is actuated. The basis of its mechanical structure is depicted in figure 3.

The motor torque produces an angular acceleration of the rotating wheel which generates, thanks to the dynamic coupling between coordinates, a torque acting on the pendulum's passive joint; therefore this passive 
TABLE I: Description of dynamic parameters of the inertia wheel inverted pendulum

\begin{tabular}{|c|c|c|c|}
\hline Parameter & Description & Value & Unit \\
\hline \hline$m_{1}$ & body mass & 3.228 & $\mathrm{~kg}$ \\
\hline$m_{2}$ & wheel mass & 0.86422 & $\mathrm{~kg}$ \\
\hline$I_{1}$ & body inertia & $3.042 \times 10^{-2}$ & $\mathrm{~kg} \mathrm{\textrm {m } ^ { 2 }}$ \\
\hline$I_{2}$ & wheel inertia & $7.986 \times 10^{-4}$ & $\mathrm{~kg} \mathrm{~m}$ \\
\hline$l_{1}$ & body CoM position & $6.354 \times 10^{-4}$ & $\mathrm{~m}$ \\
\hline$l_{2}$ & wheel CoM position & $52 \times 10^{-3}$ & $\mathrm{~m}$ \\
\hline
\end{tabular}

joint can be controlled through the acceleration of the inertia wheel.

The goal of the proposed method is to generate stable limit cycles on both coordinates using only input $u$, the applied torque on the inertia wheel. The angular position $\theta_{1}$ of the pendulum with respect to the vertical will be controlled by the first tracking controller whereas the angular velocity of the wheel will be controlled by the second controller.

\section{A. Dynamic model of the system}

The nonlinear dynamic model [16] of the plant is obtained using Lagrange formulation [20], and is given by:

$$
\begin{aligned}
\left(I_{1}+I_{2}\right) \ddot{\theta}_{1}+I_{2} \ddot{\theta}_{2}-\overline{m l} g \sin \theta_{1} & =0 \\
I_{2}\left(\ddot{\theta}_{1}+\ddot{\theta}_{2}\right) & =u
\end{aligned}
$$

where $I_{1}, I_{2}$ are respectively the moments of inertia of the beam and the wheel. $u$ is the torque generated by the motor acting on the inertia wheel. $\overline{m l}=m_{1} l_{1}+m_{2} l_{2}$ with $m_{1}$ and $m_{2}$ being the masses of the pendulum and the inertia wheel. $l_{1}, l_{2}$ are distances from origin $O$ (cf. Fig. 3) to the gravity centers of the pendulum and the rotating mass (respectively).

Table I summarizes the dynamic parameters of the inertia wheel inverted pendulum.

\section{B. Numerical simulations}

Reference trajectories are generated for the unactuated coordinate $\theta_{1}$ as presented in section II-B. The trajectories amplitude is $2 A=6^{\circ}$ and period is $\tau=2 \mathrm{~s}$.

Numerical simulations are performed using Matlab/Simulink software of MathWorks. It is worth noting that the dynamic model (14)-(15) is used to simulate the dynamic behavior of the system, it is not used in the controller design.

The following control design parameters were used: $\alpha_{1}=-100, \alpha_{2}=50$, the first controller gains $K_{p 1}=200$, $K_{i 1}=0$ and $K_{d 1}=100$, the second controller gains $K_{p 2}=K_{i 2}=0$ and $K_{d 2}=1$. However, as the inertia wheel is not limited by mechanical stops when rotating around its axis, its angular position does not matter. Consequently it is worth interesting only in its velocity. The desired state, reduced to wheel velocity $\dot{\theta}_{2}^{d}$, is set to 0 . The sampling frequency is set to $150 \mathrm{~Hz}$ which is a reasonable value for real-time implementation.
During this simulation, whose results are depicted in Fig. 4, a disturbing torque is applied to the pendulum beam at time $t=15$ seconds of intensity $0.5 \mathrm{Nm}$. The convergence to a stable limit cycle can clearly be observed on the phase portrait of the pendulum angle $\theta_{1}$. The controller reacts immediatly to the disturbance as we can see a spike in control input (cf. Fig. 4(e)). This disturbance induces a deviation from the reference trajectories of the pendulum angle position and velocity which is immediatly compensated. Despite the big deviation of the inertia wheel velocity oscillations at the beginning of the simulation due to non zero initial conditions and just after the disturbance, the second controller succesfully brings back the inertia wheel velocity trajectory to the limit cycle.

\section{Real-time experiments}

In this section experimental results are presented with some implementation issues. It starts with a description of the inverted pendulum testbed, then the obtained results are presented.

1) Experimental platform: Real time experiments are performed on the inertia wheel inverted pendulum testbed (shown in Fig. 5) designed and developed at our laboratory ${ }^{1}$. The pendulum angle $\theta_{1}$ is constrained to remain within the interval $\left[-10^{\circ}, 10^{\circ}\right]$ due to mechanical stops. The actuator of the system is a Maxon ECpowermax $30 \mathrm{DC}$ motor, equipped with an incremental encoder, allowing the measurement in real-time of the inertia wheel angular position. In order to measure the angle of the pendulum with respect to the vertical, the system is equipped with an inclinometer FAS-G of Micro strain. The system is controlled with a computer equipped with a $2.4 \mathrm{GHz}$ Intel processor. The control approach is implemented using $\mathrm{C}++$ language, and the whole system is running under Ardence RTX real-time OS.

2) Obtained results: Real-time experiments are caried out thanks to the experimental testbed described in previous section. Notice that the controller design parameters used for this experiment are different from those used in simulation. The first controller parameters are $\alpha_{1}=-150$ and gains $K_{p 1}=70, K_{i 1}=0$ and $K_{d 1}=12$. The Second controller parameters are $\alpha_{2}=70$ and gains $K_{p 2}=0, K_{i 2}=0$ and $K_{d 2}=1$. Noise filtering was performed through the use of an alpha-beta filter [21] to correctly estimate the pendulum acceleration used in equation (9).

In this experiment, external disturbances are introduced by pushing the pendulum body in a punctual fashion. Those external disturbances are introduced at approximately $t=12 \mathrm{~s}$ and $t=23 \mathrm{~s}$.

Fig. 6 shows the overall results. Noise in Measurement is observable on all plots, particularily on the pendulum velocity $\dot{\theta}_{1}$ in Fig. $6(\mathrm{~b})$. The effect of the external

\footnotetext{
${ }^{1}$ LIRMM: http: //www. lirmm.fr
} 


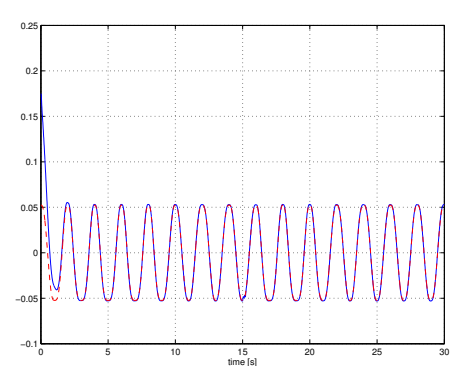

(a) pendulum position $\theta_{1}[\mathrm{rad}]$ real position (solid line) and desired position (dashed line).

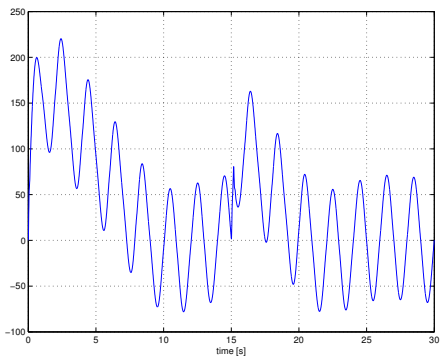

(d) inertia wheel velocity $\dot{\theta}_{2}\left[\mathrm{rad} \cdot \mathrm{s}^{-1}\right]$.

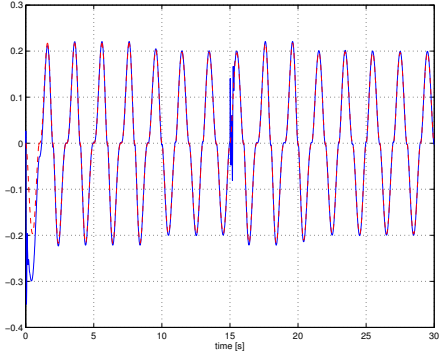

(b) pendulum

velocity $\dot{\theta}_{1}\left[\mathrm{rad} \cdot \mathrm{s}^{-1}\right]$ real velocity (solid line) and desired velocity (dashed line).

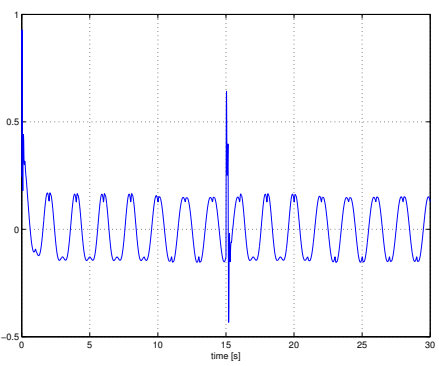

(e) control input $u[\mathrm{~N} \cdot \mathrm{m}]$ versus time.

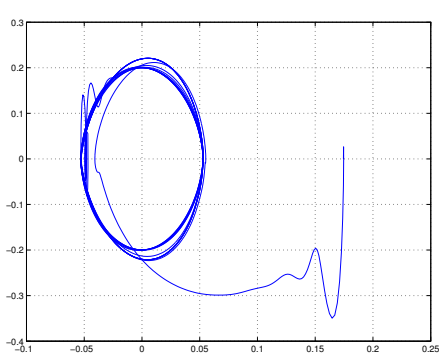

(c) phase portrait of the pendulum $\left(\theta_{1}[\mathrm{rad}], \dot{\theta}_{1}\left[\mathrm{rad} \cdot \mathrm{s}^{-1}\right]\right)$.

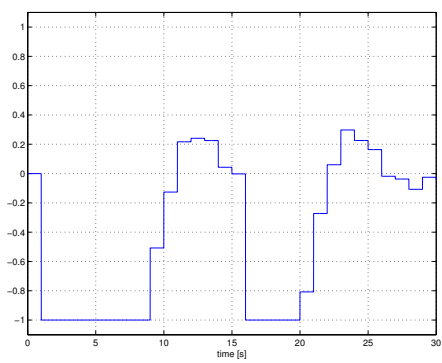

(f) evolution of parameter $p[-]$.

Fig. 4: Simulation results. A punctual external disturbance is introduced as a torque applied at time $t=15 s$ to the pendulum beam.

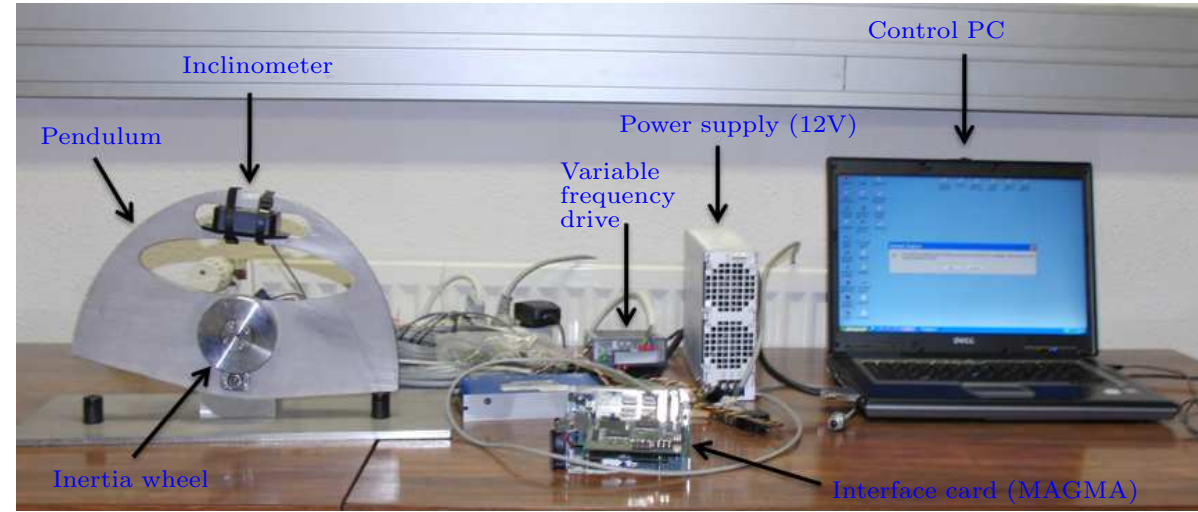

Fig. 5: Inertia wheel inverted pendulum experimental testbed

disturbances can clearly be observed on the pendulum trajectories in Fig. 6(a), and the control input in Fig. 6(e). The tracking controller brings back the pendulum position and velocity to their respective reference trajectories. The convergence to a stable limit cycle is clearly visible on the phase portrait of the pendulum shown on Fig. 6(c). The evolution the inertia wheel velocity can be observed on Fig. 6(d) The introduced disturbances induce a shift in the inertia wheel velocity trajectory cycle. However thanks to the second controller reaction, which is observable in Fig. 6(f), the inertia wheel velocity converges back to the limit cycle in few periods.

\section{CONClusion AND Future WORK}

In this paper, a dual model-free controller is proposed to deal with control of underactuated mechanical systems for stable limit cycles generation. This method inherits the advantages of model-free control: mathematical modelling of the controlled system is not required and no complex parameters identification is needed. To illustrate the effectiveness of the proposed control method, it is applied on the inertia wheel inverted pendulum. Numerical simulations and real time experimentations show the effectiveness of the proposed control scheme as well as its robustness towards external disturbances. Our future work will be focused on the optimization of the tuning parameters of the two controllers, it can also include the generalization of the proposed method to underactuated systems with more than one degree of unactuation. 


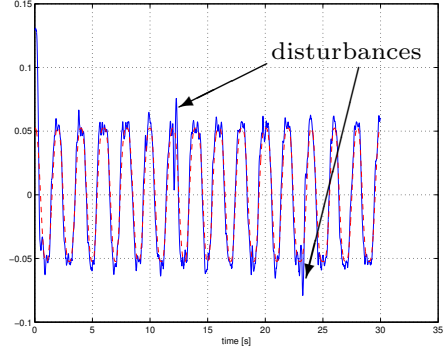

(a) pendulum position $\theta_{1}[\mathrm{rad}] \mathrm{real}$ position (solid line) and desired position (dashed line).

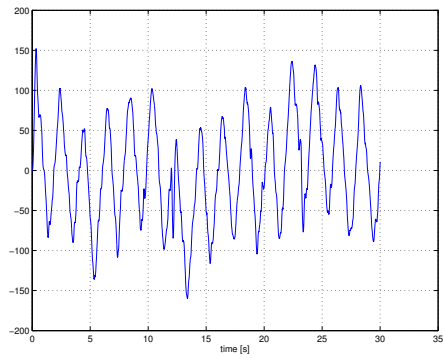

(d) inertia wheel velocity $\dot{\theta}_{2}\left[\mathrm{rad} \cdot \mathrm{s}^{-1}\right]$.

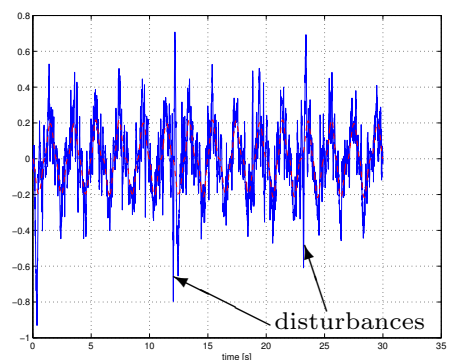

(b) pendulum velocity $\dot{\theta}_{1}\left[\mathrm{rad} \cdot \mathrm{s}^{-1}\right.$ real velocity (solid line) and desired velocity (dashed line).

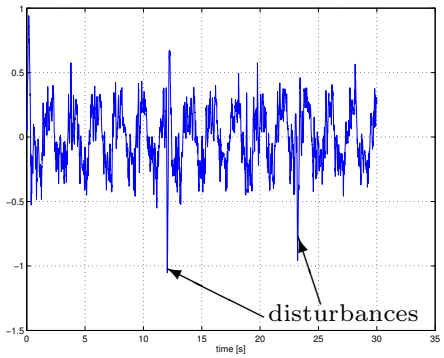

(e) control input $u[\mathrm{~N} \cdot \mathrm{m}]$ versus time.

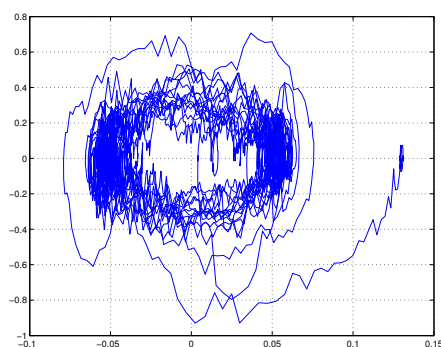

(c) phase portrait of the pendulum $\left(\theta_{1}[\mathrm{rad}], \dot{\theta}_{1}\left[\mathrm{rad} \cdot \mathrm{s}^{-1}\right]\right)$.

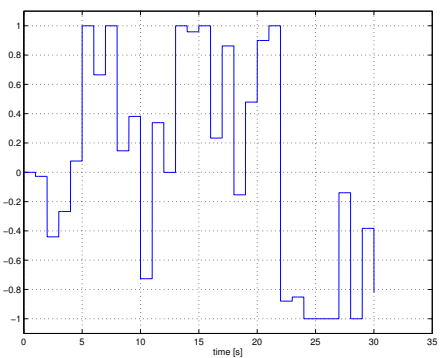

(f) evolution of parameter $p[-]$.

Fig. 6: Real-time results. Two external disturbances are introduced at $t=12 \mathrm{~s}$ and $t=23 \mathrm{~s}$ on the pendulum beam.

\section{REFERENCES}

[1] M. Fliess, C. Join, M. Mboup, and H. Sira Ramirez, "Vers une commande multivariable sans modèle," in Conférence internationale francophone d'automatique (CIFA 2006), Bordeaux, France, 2006.

[2] R. Bourdais, M. Fliess, C. Join, and W. Perruquetti, "Towards a model-free output tracking of switched nonlinear systems," in NOLCOS 2007 - 7th IFAC Symposium on Nonlinear Control Systems, Pretoria, South Africa, 2007.

[3] M. Fliess, C. Join, and H. Sira Ramirez, "Non-linear estimation is easy," Int. J. Modelling Identification and Control, vol. 4, pp. $12-27,2008$.

[4] M. Mboup, C. Join, and M. Fliess, "Numerical differentiation with annihilators in noisy environment," Numerical Algorithms, vol. 50, pp. 439-467, 2009.

[5] B. D'Andrea Novel, M. Fliess, C. Join, H. Mounier, and B. Steux, "A mathematical explanation via "intelligent" PID controllers of the strange ubiquity of PIDs," in 18th Mediterranean Conference on Control and Automation, MED'10, Marrakech Morocco, 2010.

[6] P.-A. Gédouin, C. Join, E. Delaleau, J.-M. Bourgeot, S. Arbab Chirani, and S. Calloch, "A new control strategy for shape memory alloys actuators," in 8th European Symposium on Martensitic Transformations, Prague, Czech Republic, 2009.

[7] C. Join, G. Robert, and M. Fliess, "Model-free based water level control for hydroelectric power plants," in IFAC Conference on Control Methodologies and Tecnologies for Energy Efficiency, CMTEE, Vilamoura, Portugal, 2010.

[8] L. Michel, C. Join, M. Fliess, P. Sicard, and A. Chériti, "Modelfree control of dc/dc converters," in 12th IEEE Workshop on Control and Modeling for Power Electronics (COMPEL), Boulder, Colorado United States, 2010.

[9] M. Fliess and C. Join, "Model-free control and intelligent PID controllers: towards a possible trivialization of nonlinear control?" in 15th IFAC Symposium on System Identification (SYSID 2009), Saint-Malo, France, 2009.

[10] S. Riachy, M. Fliess, C. Join, and J.-P. Barbot, "Vers une simplification de la commande non linéaire : l'exemple d'un avion à décollage vertical," in Sixième Conférence Internationale Francophone d'Automatique, CIFA 2010, Nancy, France, 2010.
[11] M. W. Spong, P. Corke, and R. Lozano, "Nonlinear control of the inertia wheel pendulum," Automatica, vol. 37, pp. 1845$1851,1999$.

[12] R. Ortega, M. Spong, F. Gómez-Estern, and G. Blankenstein, "Stabilization of a class of underactuated mechanical systems via interconnection and damping assignment," IEEE Transactions on Automatic Control, vol. 48, no. 8, pp. 1218 -1233, 2002.

[13] C. Aguilar-Ibanez, O. Gutierrez F, and M. Suarez Castanon, "Stabilization of the strongly damping inertia wheel pendulum by a nested saturation functions," in Proc. of American Control Conference, 2008, pp. 3434 - 3439.

[14] L. Freidovich, P. La Hera, U. Mettin, A. Robertsson, A. Shiriaev, and R. Johansson, "Shaping stable periodic motions of inertia wheel pendulum: Theory and experiments," Asian Journal of Control, vol. 11, no. 5, pp. 548-556, 2009.

[15] N. Qaiser, S. B. Tariq, N. Haq, and T. Aziz, "A novel nonlinear implicit sliding surface controller design for inertia wheel pendulum," in Proceedings of the 10th WSEAS international conference on Robotics, control and manufacturing technology, ser. ROCOM'10. Stevens Point, Wisconsin, USA: World Scientific and Engineering Academy and Society (WSEAS), 2010, pp. 21-25.

[16] S. Andary, A. Chemori, and S. Krut, "Control of the Underactuated Inertia Wheel Inverted Pendulum for Stable Limit Cycle Generation," RSJ Advanced Robotics, vol. 23, no. 15, pp. 1999-2014, 122009.

[17] S. Andary, A. Chemori, and S. Krut, "Estimation-based disturbance rejection in control for limit cycle generation on inertia wheel inverted pendulum testbed," in IROS'09: Proceedings of the 2009 IEEE/RSJ international conference on Intelligent robots and systems. Piscataway, NJ, USA: IEEE Press, 2009, pp. 1302-1307.

[18] L. Sciavicco and B. Siciliano, Modeling and control of robot manipulators. New York: McGraw Hill, 1996.

[19] M. W. Spong, "Underactuated mechanical systems," in Control Problems in Robotics and Automation. Springer-Verlag, 1998.

[20] M. Spong and M. Vidyasagar, Robot Dynamics and Control. New York, USA: John Wiley and Sons, 1989.

[21] R. Penoyer, "The alpha-beta filter," C Users J., vol. 11, pp. 73-86, 1993 